\title{
Erosion of Gastric Band Tubing Presenting as Port-Site Cellulitis
}

\author{
Melissa Felinski, DO, Kulvinder S. Bajwa, MD, Shinil K. Shah, DO \\ Department of Surgery, McGovern Medical School at UT Health, Houston, Texas, USA (all authors). \\ Michael E. Debakey Institute for Comparative Cardiovascular Science and Biomedical Devices, Texas A\&M University, \\ College Station, Texas, USA (Dr. Shah).
}

\begin{abstract}
Introduction: Long-term outcomes of laparoscopic adjustable gastric band (LAGB) placement have shown suboptimal excess weight loss and higher than expected device-related complications.

Case Description: We report a 45-year-old woman in whom the adjustable gastric band tubing eroded into the stomach, causing a port-site infection.

Conclusion: Because of the number of LAGB procedures performed previously and the incidence of band-related complications, surgeons must be able to recognize and manage these complications. Erosion of the gastric band or the connection tubing or both should be included in the differential diagnosis for patients presenting with a port-site infection.
\end{abstract}

Key Words: Complications, Erosion, Gastric band.

Citation Felinski M, Bajwa KS, Shah SK. Erosion of gastric band tubing presenting as port-site cellulitis. CRSLS e2017.00022. DOI: 10.4293/CRSLS.2017.00022.

Copyright (c) 2017 by SLS, Society of Laparoendoscopic Surgeons. This is an open-access article distributed under the terms of the Creative Commons Attribution-Noncommercial-ShareAlike 3.0 Unported license, which permits unrestricted noncommercial use, distribution, and reproduction in any medium, provided the original author and source are credited.

Disclosures: None

Address correspondence to: Shinil K. Shah, DO, Department of Surgery, McGovern Medical School at UT Health, 6431 Fannin Street, Houston, TX 77030, USA.

Telephone: 713-500-7277. Fax: 713-383-3708 E-mail: shinil.k.shah@uth.tmc.edu

\section{INTRODUCTION}

Although once a popular procedure in patients desiring bariatric surgery, laparoscopic adjustable gastric band (LAGB) placement has been steadily declining. Although the simplicity of the device, ease of placement, reversibility, and safety profile were initially appealing, the LAGB has been criticized as less effective, with a higher rate of revision than other weight loss procedures. Various studies reviewing long-term outcomes found that the Rouxen-y gastric bypass and sleeve gastrectomy are superior to the LAGB in durable weight loss and improvement of obesity-related comorbidities. ${ }^{1-4}$

In addition to suboptimal results, long-term follow-up has shown that the LAGB is associated with device-related complications, including band slippage, erosion, dilation of the gastric pouch or esophagus, band and port leaks, and infection. Studies reveal a 12-48\% complication rate, $, 5,6$ with up to $50 \%$ of patients with the band requiring reoperation at 10 years. ${ }^{1,5}$

Erosion is recognized as one of the most serious complications after LAGB. It is a relatively rare, yet well-reported complication, with an overall incidence rate of $1-3 \% .5,7,8$ Erosion is more commonly associated with the band itself, but it may also rarely occur as a result of the connection tubing. We present a case of gastric erosion caused by the LAGB connection tubing.

\section{CASE REPORT}

The patient was a 45-year-old woman with a history of morbid obesity who presented with a 2 -week history of pain and swelling at the site of her LAGB port. She had undergone placement of an LAGB (Lap Band; Allergan, Irvine, California, USA) $\sim 4.5$ years before presenting with successful weight loss (body mass index, 22). Four months before her current presentation, she experienced 
mild reflux secondary to band slippage and had $8 \mathrm{~mL}$ of fluid removed from the band. She had $4 \mathrm{~mL}$ of fluid replaced the following month. At the time of band adjustment, the patient reported difficulty accessing the port, requiring multiple attempts. Since then, she had been having intermittent pain at the site of the port. Over the next several weeks she developed erythema, swelling, and increasing tenderness to the area. Her symptoms failed to improve after self-treatment with a short course of oral antibiotics. On admission, she was afebrile, with erythema at the LAGB port site and a normal white blood cell count. Computed tomographic imaging demonstrated stranding around the LAGB port, with thickening of the rectus abdominis (Figure 1A), and the band tubing appeared to have eroded into the lumen of the stomach (Figure 1B).

Given the concern for erosion, the patient was taken to the operating room for diagnostic laparoscopy, esophagogastroduodenoscopy, and possible LAGB removal. There were significant adhesions of the stomach and omentum to the anterior abdominal wall at the site of suspected erosion. Given the extent of inflammatory response and pre-existing adhesions, minimal dissection was performed in this area, as the site of erosion appeared to be self-sealed. The LAGB was noted to have slipped. Upper endoscopy confirmed that the LAGB connection tubing had eroded through the antrum of the stomach (Figure 2A and $\mathbf{B}$ ) and corresponded to the site of inflammation seen on laparoscopic examination. The LAGB and associated capsule were carefully dissected, and the band was unbuckled and removed from around the stomach. The LAGB tubing was cut and the band was removed. No evidence of an air leak was seen on repeat endoscopy. The subcutaneous port and associated connection tubing were removed via an incision directly over the port. Murky fluid was encountered and sent for gram stain and culture. The port-site incision was closed over a vessel loop with Monocryl sutures (Ethicon, Somerville, New Jersey, USA). An upper gastrointestinal swallow study on postoperative day 1 revealed no evidence of leak or contrast extravasation. The patient was started on a clear liquid diet and was discharged without complication on postoperative day 3. At clinic follow-up, she was doing well with no complaints.

\section{DISCUSSION}

Morbid obesity is a chronic disease that can be effectively treated with bariatric surgery. One of the surgical options, the LAGB, had an enormous rise in popularity because of its relatively low perioperative morbidity, quick recovery, adjustability, and reversibility in comparison to other bari-
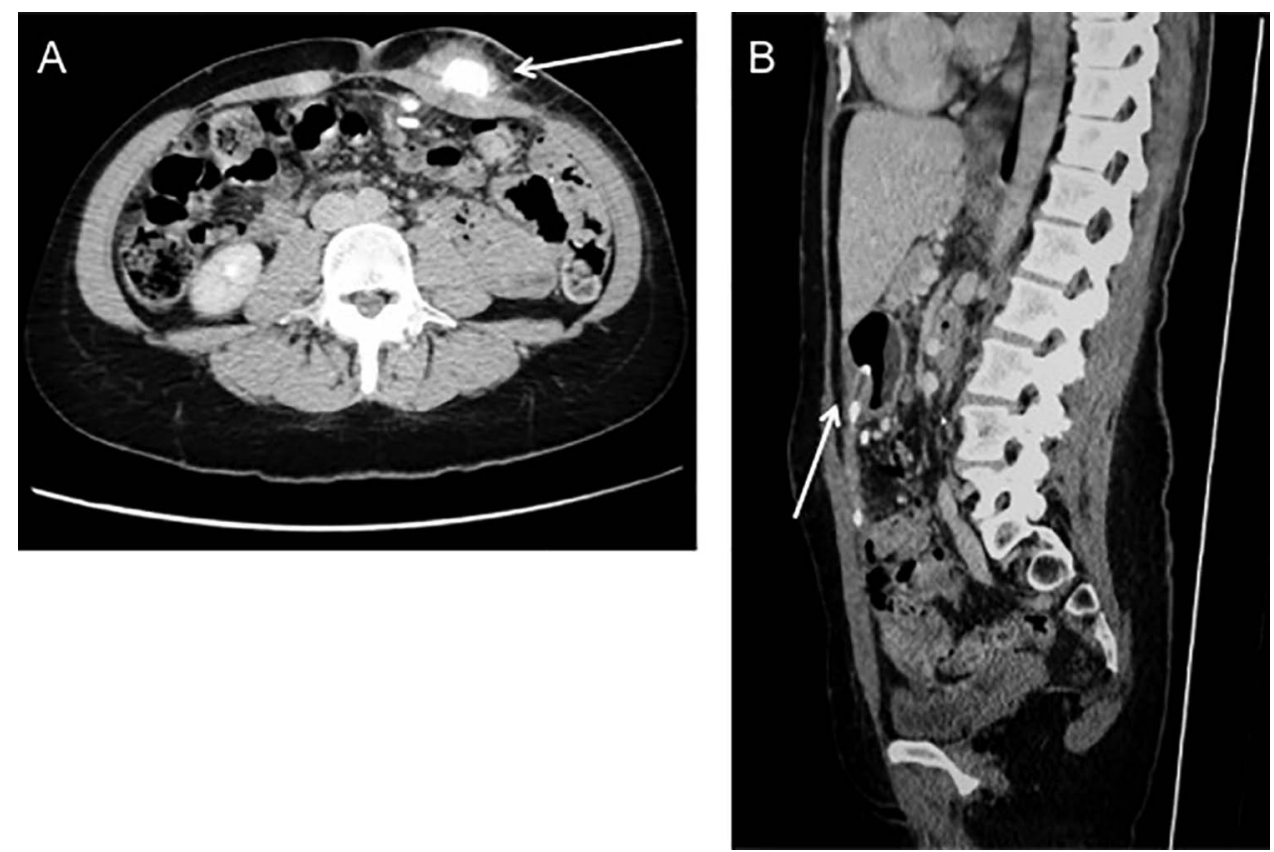

Figure 1. CT scan demonstrates significant stranding around the subcutaneous port (arrow), with inflammatory changes seen around the rectus abdominis (A). Port tubing (arrow) appears to have eroded into the lumen of the stomach (B). 

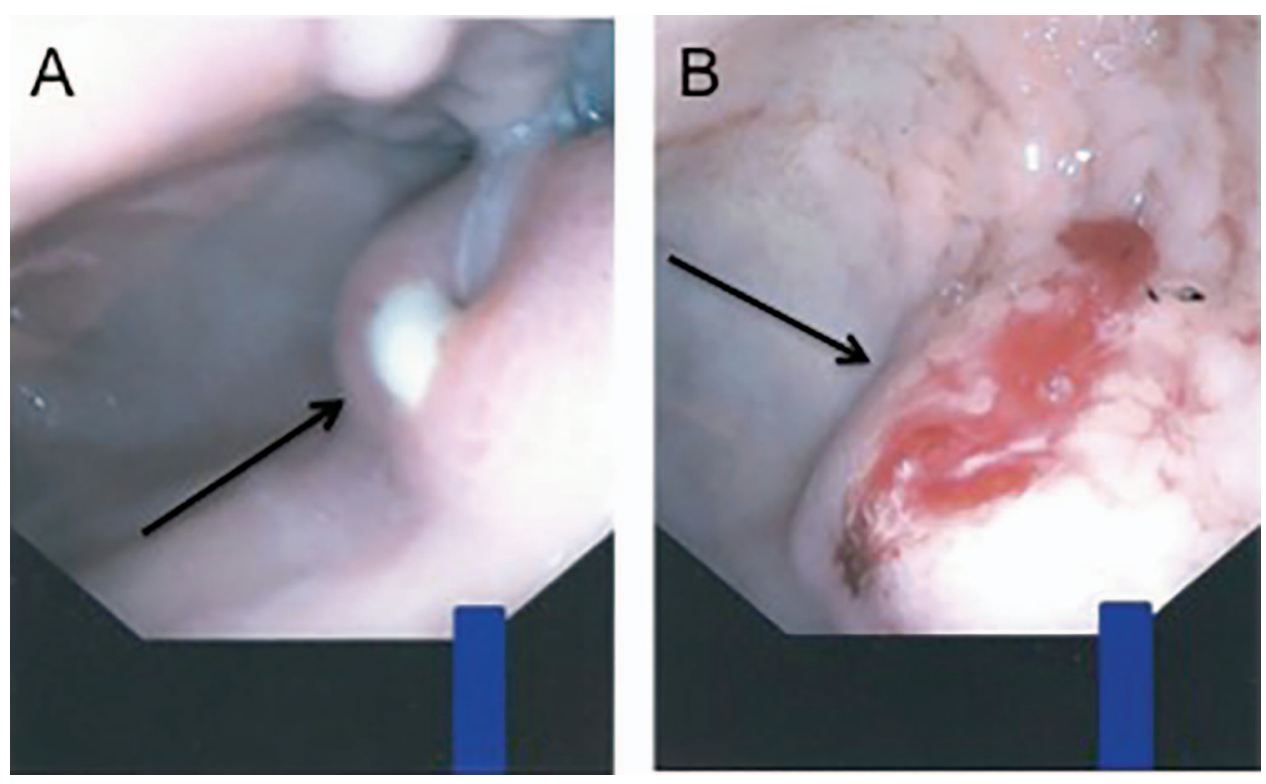

Figure 2. Intraoperative endoscopy confirms intraluminal erosion of the gastric band tubing (arrows) before (A) and after (B) removal.

atric procedures. Patients and surgeons alike were satisfied with its ease of use, associated weight loss, and improvement in obesity-related comorbidities. Typically, a motivated, informed patient with rigorous follow-up could expect to lose $46-48 \%$ of excess body weight, with a peak weight loss at 2-3 years after LAGB. ${ }^{9}$

However, as the LAGB failed to live up to expectations beyond the initial short-term, there has been a considerable decrease in its use in favor of Roux-en-y gastric bypass and sleeve gastrectomy. The number of LAGB procedures performed has fallen from its peak in 2008 at $42 \%$ of all weight loss procedures, ${ }^{10} 35.4 \%$ in 2011, and $5.7 \%$ in $2015 .{ }^{11}$ Various studies reviewing the long-term outcomes up to 10-15 years after LAGB demonstrate disappointing excess weight loss, weight regain, and reappearance of obesity-related conditions. Snyder et $\mathrm{al}^{6}$ showed that the failure to achieve a satisfactory excessive weight loss in the first several years after surgery range from $10-20 \%$ with an even greater failure rate occurring when evaluated long-term. Likewise, multiple studies have reported better long-term weight loss and resolution of comorbidities with other bariatric procedures ${ }^{6,7}$ when compared to LAGB.

In addition to less effective and durable weight loss, the LAGB is associated with a number of complications associated with the mechanical device. An overall devicerelated complication rate of $12-48 \%$ with a reoperation rate ranging from $10-34 \%$ has been reported in the literature. ${ }^{6}$ Other series have reported that $40-50 \%$ of band recipients undergo additional surgery over the 10-year follow-up period. ${ }^{1,5}$ Aarts et al12 published a study of 201 patients who underwent LAGB, of which $53 \%$ had the band removed or converted to another bariatric surgery approach because of insufficient weight loss or complications. In addition, in this series, less than $25 \%$ of patients still had the band in place 14 years after the original placement.

Complications of the LAGB include band slippage, erosion, pouch or esophageal dilation, port-site infection, and leakage of the band, port, or tubing. Band erosion may be considered one of the more serious complications of LAGB, with an incidence ranging from 1 to $3 \% .{ }^{6} \mathrm{Al}-$ though most erosions occur where the gastric wall is in contact with the band, erosion of the connection tubing into the intestine and neighboring structures, such as the colon, celiac axis, and renal hilum, is a much rarer, yet reported complication. ${ }^{13-19}$ Most authors report a median time of 12-24 months from band placement to erosion, but it may occur more than 10 years after LAGB placement. ${ }^{20,21}$ Because the erosion tends to develop gradually over time, ${ }^{21}$ most cases are nonurgent. It is speculated that this is a result of the tissue capsule that forms around the band, preventing free perforation and leakage.

Diagnosis may be delayed, as patients can present with subtle, nonspecific symptoms, if any at all. Symptoms include epigastric pain, nausea, vomiting, loss of satiety, port-site infections, intra-abdominal abscess, or rarely, generalized peritonitis. The patient in the case description 
was given a short course of self-administered oral antibiotics for a presumed port-site infection secondary to manipulation during band fill adjustment. It is important to note that late or recurrent port-site infections could be related to gastric, duodenal, or colonic erosion until proven otherwise. Up to half of patients remain asymptomatic, ${ }^{7}$ making erosion difficult to diagnosis even for those with experience in bariatric and gastrointestinal surgery.

Clinical suspicion of gastric band or connection tubing erosion should be investigated further. Although upper endoscopy is the modality of choice for the diagnosis of an intragastric band or connection tubing erosion, less invasive methods, such as computed tomographic (CT) imaging or upper gastrointestinal series may be used when organs other than the stomach are suspected to be involved. Once the diagnosis is confirmed, the management of a patient with an eroded gastric band or connection tubing depends on the site of erosion, the component of the band device involved, and the experience of the surgeon.

Surgical explantation can be performed via an open or laparoscopic approach with suture repair of the defect and omentopexy, if indicated. A minimal dissection approach should be used, and attempts to unroof the site of erosion should be avoided if the perforation appears to be self-sealed. An intra-operative leak test should be performed to confirm the absence of a leak. Although the routine use of drains has not been noted to decrease the incidence of intra-abdominal abscesses and can lead to drain-related morbidity, drain placement should be considered in difficult or high-risk cases. Endoscopic band removal is a less invasive means that can be used when the buckle of the band is inside the stomach but may be more technically demanding for a novice endoscopist. During removal, ensuring complete retrieval of the band and all its components is imperative. All explanted parts should be carefully examined and pieced together to avoid retention of a foreign object.

\section{CONCLUSION}

Perioperative complications are minimal compared to other bariatric procedures, but LAGB has a relatively high reoperation rate because of failure to achieve the desired weight loss or complications related to the band or it components. Erosion of the gastric band or connection tubing or both is a rare complication, but should be included in the differential diagnosis for a patient presenting with a port-site infection. As the long-term complica- tions of gastric bands are realized, the historic volume of procedures necessitates that the surgeon be able to recognize and manage these complications.

\section{References:}

1. Angrisani L, Cutolo PP, Formisano G, Nosso G, Vitolo G. Laparoscopic adjustable gastric banding versus Roux-en-Y gastric bypass: 10-year results of a prospective, randomized trial. Surg Obes Relat Dis. 2013;9:405-413.

2. Caiazzo R, Lassailly G, Leteurtre E, et al. Roux-en-Y gastric bypass versus adjustable gastric banding to reduce nonalcoholic fatty liver disease: a 5-year controlled longitudinal study. Ann Surg. 2014;260:893-898; discussion 898-899.

3. Himpens J, Dapri G, Cadiere GB. A prospective randomized study between laparoscopic gastric banding and laparoscopic isolated sleeve gastrectomy: results after 1 and 3 years. Obes Surg. 2006;16:1450-1456.

4. Puzziferri N, Roshek TB 3rd, Mayo HG, Gallagher R, Belle $\mathrm{SH}$, Livingston EH. Long-term follow-up after bariatric surgery: a systematic review. JAMA. 2014;312:934-942.

5. O'Brien PE, MacDonald L, Anderson M, Brennan L, Brown WA. Long-term outcomes after bariatric surgery: fifteen-year follow-up of adjustable gastric banding and a systematic review of the bariatric surgical literature. Ann Surg. 2013;257:87-94.

6. Snyder B, Wilson T, Mehta S, et al. Past, present, and future: Critical analysis of use of gastric bands in obese patients. Diabetes Metab Syndr Obes. 2010;3:55-65.

7. Owers C, Ackroyd R. A study examining the complications associated with gastric banding. Obes Surg. 2013;23:56-59.

8. Quadri P, Gonzalez-Heredia R, Masrur M, Sanchez-Johnsen L, Elli EF. Management of laparoscopic adjustable gastric band erosion. Surg Endosc. 2017;31(4):1505-1512.

9. Brethauer SA, Chand B, Schauer PR. Risks and benefits of bariatric surgery: current evidence. Cleve Clin J Med. 2006;73: 993-1007.

10. Buchwald H, Oien DM. Metabolic/bariatric surgery Worldwide 2008. Obes Surg. 2009;19:1605-1611.

11. American Society of Metabolic and Bariatric Surgeons. Estimate of bariatric surgery numbers, 2011-2015. Gainesville, FL: ASMBS, 2016 https://asmbs.org/resources/estimate-of-bariatricsurgery-numbers. Accessed January 26, 2017.

12. Aarts EO, Dogan K, Koehestanie P, Aufenacker TJ, Janssen IM, Berends FJ. Long-term results after laparoscopic adjustable gastric banding: a mean fourteen year follow-up study. Surg Obes Relat Dis. 2014;10:633-640.

13. Manatakis DK, Terzis I, Kyriazanos ID, et al. Simultaneous gastric and duodenal erosions due to adjustable gastric banding for morbid obesity. Case Rep Surg. 2014;2014:146980. 
14. Cintolo JA, Levine MS, Huang S, Dumon K. Intraluminal erosion of laparoscopic gastric band tubing into duodenum with recurrent port-site infections. J Laparoendosc Adv Surg Tech Part A. 2012;22:591-594.

15. Al-Bahri S, Gonzalvo JP, Murr M. Simultaneous gastric and colonic band erosion presenting as lower gastrointestinal bleeding. Surg Obes Relat Dis. 2017;13:538-539.

16. Strahan A, Aseervatham R. Laparoscopic adjustable gastric band tubing erosion into large bowel. ANZ J Surg. Jan 12, 2015. doi: 10.1111/ans.12963. [Epub ahead of print]

17. Tan LB, So JB, Shabbir A. Connection tubing causing small bowel obstruction and colonic erosion as a rare complication after laparoscopic gastric banding: a case report. J Med Case Rep. 2012;6:9.

18. Iqbal M, Manjunath S, Seenath M, Khan A. Massive upper gastrointestinal hemorrhage: an unusual presentation after laparoscopic adjustable gastric banding due to erosion into the celiac axis. Obes Surg. 2008;18:759-760.

19. Sneijder R, Cense HA, Hunfeld M, Breederveld RS. A rare complication after laparoscopic gastric banding: connectingtube penetration into the hilus of the kidney. Obes Surg. 2009; 19:531-533.

20. Brown WA, Egberts KJ, Franke-Richard D, Thodiyil P, Anderson ML, O'Brien PE. Erosions after laparoscopic adjustable gastric banding: diagnosis and management. Ann Surg. 2013; 257:1047-1052.

21. Egberts K, Brown WA, O'Brien PE. Systematic review of erosion after laparoscopic adjustable gastric banding. Obes Surg. 2011;21:1272-1279. 